\title{
Path loss prediction models for Corridor propagation at $24 \mathrm{GHz}$
}

\author{
Femi-Jemilohun Oladunni .J and Walker Stuart .D \\ School of Computer Science and Electronic Engineering, University of Essex, United Kingdom; \\ ojfemi@essex.ac.uk
}

\begin{abstract}
Mm-wave bands have recently become major options for short-range, high speed communication systems especially in the indoor wireless local area networks (WLANs). The pathloss prediction model is one of the metric parameters for determining the system effectiveness and performance in wireless indoor propagation. The channel characterization of $24 \mathrm{GHz}$ band in corridor propagation through extensive field strength measurements in real time application was conducted in this work. The results were used to derive the path loss equation for corridor propagation at this spectrum band based on log-distance path loss model and log-normal shadowing model. The pathloss realized falls within the estimated values in such scenario, it is therefore concluded that the predicted mathematical model for the described environment is accurate. Also the predicted pathloss which is lower than the free space propagation path loss results in aggregate high data rate, hence improved system performance is achieved.
\end{abstract}

Keywords: Pathloss, millimeter wave, multipath, Quality of Service, and fading

\section{Introduction}

The ever increasing supply of, and demand for, broadband multimedia to match up with the ever increasing capacity of wireless networks, had led to wireless transfer demand that is far beyond what the current bands in the Industrial, Scientific and Medical (ISM) and Unlicensed National Information Infrastructure (UNII) bands can accommodate. A way out is to resort to the millimeter wave ( $\mathrm{mmw}$ ) bands. Also the high data rates intended for $4 \mathrm{G}$ infrastructures will require the use of unlicensed spectrum with high and sufficient bandwidth to accommodate such huge capacities [1]. Mm-wave bands such as $24 \mathrm{GHz}$ and $60 \mathrm{GHz}$ have recently become the major options for short-range, high speed communication systems especially in the indoor wireless local area networks (WLANs). These bands offer the gigabit per second (Gb/s) throughput required by multimedia consumer-oriented applications. For the dramatic growth in appreciation and application of wireless communications, high quality of service (QoS), increase in the system reliability and capacity are inevitable for seamless communication systems. To 
achieve this in mobile radio channel, the technical challenges peculiar to $\mathrm{mmw}$ such as multipath fading, polarization mismatch, and co-location interference must be adequately addressed [2]

Also, as e-commerce is becoming more widely used, it is expected that good internet services are available to end-users as they commute from place to place. In this scenario, as mobile terminals move from one office to the other through corridor and the like, wireless services should retain their high-throughput during the transit events [3]. The task of modelling radio propagation at hallway and tunnels is enormous. Among the various channel modelling techniques, ray tracing is well accepted [4-6]. It is noted that propagation in corridors and hallways suffers less losses than free space loss, on the contrary propagation through walls and floors incurs higher losses than free space loss [7].

This is similar to the experience in the corridor propagation as revealed by the results of the experimental work. This work carried out extensive signal strength measurements along a level four of a multi-storey building corridor. The results were used to determine the path loss exponent and standard deviation based on Log-distance path loss model and Log- normal shadowing respectively. An equation that describes the path loss of our propagation scenario was derived through numerical analysis of the results. The remainder of this paper is arranged as follows: Section two discussed the previous research works by different authors in the relevant area. The relevant background study of the topic was presented in section three, while the results and discussion of the experimental work carried out were presented in section four. Finally, the conclusion was given in section five.

\section{Related Work}

A lot of research works have been done and still on going in the area of wireless communication performances. Interest is been focused on the WLAN technology to provide the gigabits throughput required by multimedia applications, video conferencing, data streaming and many more services especially in an office environment as well campuses. In literature, there are some works on system performance evaluation and interference mitigation techniques in WLAN. However, very few of these authors have engaged physical devices in their works to realize real values. Likewise, the possibility of engaging $24 \mathrm{GHz}$ bands for wireless indoor propagation in multipath rich environment such as corridor has not been conducted by any author to the best of my knowledge. The first set of results in indoor WLAN at $24 \mathrm{GHz}$ is presented in this work. Some of the findings of the previous authors related to this work are enumerated as follows:

The overview of the newest technologies promised to deliver multi-gigabits throughput through IEEE802.11ac and IEEE802.11ad standards was carried out in [8]. The author described the channelization of physical (PHY) design, medium access control (MAC) modifications, and beamforming in the standards. In [9], the pathloss and delay characteristics of indoor radio channels from $2.4 \mathrm{GHz}$ to $24 \mathrm{GHz}$ were carefully investigated in a typical modern building. One 
particularly interesting conclusion was that delay variation increased with frequency in the Non-Line-of- Sight (NLoS) case in contrast to Line-of-Sight (LoS). In [10], the same authors used ray-tracing techniques to model, with good accuracy, 2.4 to $24 \mathrm{GHz}$ path loss in the NLoS cases. They also found that delay variation was only predicted reliably in the LoS case. A $2.4 \mathrm{GHz}$ indoor radio WLAN in a dense office environment was examined in [11]. It was concluded, inter alia, that the antenna properties can have a large impact on performance. The effects of wall materials on the attenuation of radio waves in indoor propagation up to $5 \mathrm{GHz}$ were considered in [12]. They observed that signal attenuation is differentiated by wood or concrete construction and its dependence on frequency.

From this review, it is concluded firstly that the $24 \mathrm{GHz}$ band is worth considering for the indoor environment. Secondly, we believe this present work to be the first investigation of 24 $\mathrm{GHz}$ band, high-data rate, within-building, wireless systems. Research showed that there is a logarithmic decrease relationship between the average signal power and the distance in a theoretical and measurement based propagation models. The computational complexity involved can be reduced by empirical models while prediction accuracy is increased [13]. This work is based on Log-distance path loss model and Log-normal shadowing.

\section{Basic propagation property at MMW}

The success of radio wave propagation in a building is affected by the topology, construction and materials of the building. A modern building with open-plan design containing walls with large glass windows will give added path loss of about $5 \mathrm{~dB}$ while a building made of thick stone walls with small windows and many internal solid walls will add an extra path loss of several tens of $d B$ (decibel). Some of the physical effects of wireless indoor propagation are fast decay of signals, constrained coverage by walls, and attenuation by walls, floors, furniture, and scattering of radio waves [14].

\subsection{Fresnel Zone}

It is important to maintain a radio frequency (RF) LoS between the transmitting and receiving terminals for effective long range wireless communication systems. Visual LoS is a straight line path that enables a clear sight between two terminals. In any case RF LoS requires both virtual LoS as well as a Fresnel zone that is void of obstacles to achieve optimum data transfer from one point to another. Fresnel zone is defined as the long ellipsoid path between two terminals that creates a path for RF signals. It is very essential that the Fresnel zone be freed of any obstructions such as buildings, trees, humans, as these will degrade the communication networks and reduce the range. As a rule of thumb, $60 \%$ of this zone must be cleared of obstruction. Any transmission path void of Fresnel zone is termed RF NLoS, this a typical experience in indoor propagation environment [15]. 


\subsection{Multipath and fade margins}

Multipath is the splitting of the RF signals from the transmitter along different paths. This phenomenon can be constructive when the waves travelling along different paths combined in phase at the receiver, otherwise destructive when they combine out of phase thereby cancelling out the signals. Fading is as a result of multipath, it is the difference between the normal received power and the power required for minimum acceptable performance. Greater fade margin imply less frequent occurrences of minimum performance levels, this also means that the received signal during unfaded condition is so strong that bit errors are virtually nonexistent. Severe fading due to multipath can cause a signal degradation of more than $30 \mathrm{~dB}$, which will affect the reliability of the communication links [16].

\subsection{Pathloss models}

A propagation model is a set of mathematical expressions, diagrams, and algorithms used to represent the radio characteristics of a given environment. The pathloss prediction models can be either empirical (also called statistical) or theoretical (also called deterministic), or a combination of these two. The empirical models are based on measurements, while the theoretical models deal with the fundamental principles of radio wave propagation phenomena [17]. Some existing pathloss models are listed below:

\subsubsection{Okumura-Hata Model}

This is a combination of two models developed by Masaharu Hata and Okumura. This model accuracy is high as it is based on measurements in a specific environment but it can only be used to predict the path loss of outdoor propagation. It is expressed mathematical as follows [18]:

$$
\begin{aligned}
& L_{50}(d B)=69.55+26.16 \log \left(f_{c}\right)-13.82 \log \left(h_{t e}\right)-a\left(h_{r e}\right) \\
& \quad+\left(44.9-0.55 \log \left(h_{t e}\right)\right) \log (d)
\end{aligned}
$$

Where, $L_{50}(d B)$ is the 50th percentile median pathloss, $f_{c}$ is the center frequency in megahertz, $h_{t e}$ and $h_{t e}$ are base and receiver stations antennas heights in meters respectively, $\mathrm{a}\left(h_{r e}\right)$ is a vehicular station antenna height-gain correction factor depending on the environment, and $d$ is the link distance in kilometers.

\subsubsection{Log-distance pathloss model and Log-Normal shadowing}

These are acceptable models for prediction of pathloss in an indoor/NLoS propagation environment. They show the linear relationship between the pathloss in decibel and the logarithmic variation of the transmitter and receiver separation [2]. The path loss exponent (n) on which large scale pathloss of random T-R separation depends is a function of the propagation environment while reduced value of $n$ gives lower signal loss. The value of $n$ for free space is 2, it ranges from 1.2 (waveguide effect) to 8 in general. Equations ( 2 and 3 ) depict the parametric relationship of Log-distance pathloss model. 
The different level of clustering on the signal propagation path leads to random shadowing effects. This is not accounted for in Log-distance path loss but in Log-normal shadowing as shown in (4). The average pathloss for a given transmitter and receiver distance $d$ is given by $[19,20]$ as follows:

$$
\begin{aligned}
& P L_{d} \propto\left(\frac{d}{d_{0}}\right)^{n} \\
& P L_{d B}=U L_{d 0}+10 n \log \left(\frac{d}{d_{0}}\right)
\end{aligned}
$$

Where $\mathrm{PL}$ is the average pathloss between transmitter and receiver, $U L_{d 0}$ is the reference pathloss at $d_{0}==1 \mathrm{~m}$ for indoor propagation, $\mathrm{n}$ is the pathloss exponent and $\mathrm{d}$ is the separation between transmitter and receiver in meters. (2) is modified as shown below to give the LogNormal Shadowing equation.

$$
P L_{d B}=U L_{d_{0}}+10 n \log \left(\frac{d}{d_{0}}\right)+X_{\sigma}
$$

\subsubsection{Joint Technical Committee (JTC) Model}

The mathematical representation of this model is given according to [21] [22] as:

$$
L_{\text {Total }}=A+B \log _{10}(d)+L_{f}(n)+X_{\sigma}
$$

Where $A$ is an environmental dependent fixed loss factor in $\mathrm{dB}, \mathrm{B}$ is the distance dependent loss coefficient, $\mathrm{d}$ is the separation between transmitter and receiver in meter $L_{f}$ is a floor/wall penetration loss factor in $d B, n$ is the number of floors/walls between the transmitter and receiver, and $X_{\sigma}$ is a normal (Gaussian) random variable in $\mathrm{dB}$ with zero mean and standard deviation $\sigma$ in $\mathrm{d}$.

\section{Experimental methodology}

The experimental set up shown in Fig 1 consists of the $24 \mathrm{GHz}$ point-to-point link with maximum output power of $20 \mathrm{dBm}$. It has delivery capacity of $1.4 \mathrm{Gbps}$ using the HDD in bidirectional mode at 6X64 QAM modulations scheme (highest) and is backward compatible to lower modulation scheme of QPSK through the automatic rate adaptation to accommodate low signal transmission. The feature enables a link pair to sustain up to $142.5 \mathrm{~dB}$ path loss when switched to basic QPSK modulation mode. Full duplex transmission is used with slight different carrier frequency of 24.1 and $24.2 \mathrm{GHz}$; a bandwidth of $100 \mathrm{MHz}$. The transmitting and the receiving terminals have an antenna gain of 33dBi each [23][24]. For the empirical experiment, both antennas were mounted on tripods $1.7 \mathrm{~m}$ above the floor level, and connected to PCs for signal transmission monitoring. The link was set up in the corridor as shown in Fig 2, where the signal strength measurements at eight different distance locations from $1 \mathrm{~m}-36 \mathrm{~m}$ at step of 5 were taken during propagation. 


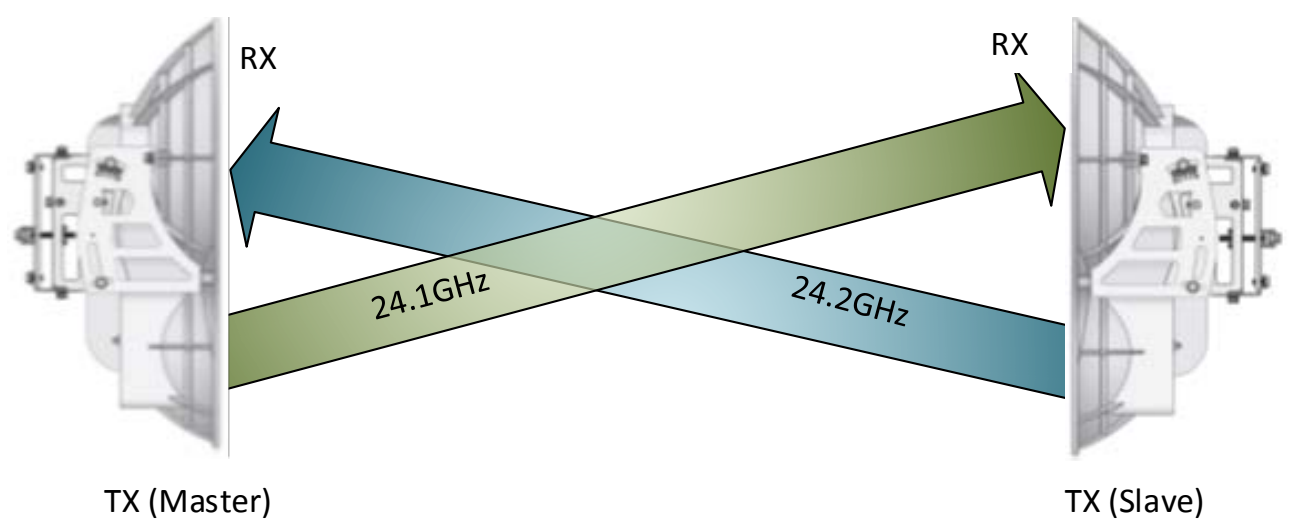

Fig.1: airFibre Ubiquiti $24 \mathrm{GHz}$ back-to-back set-up in full duplex operation

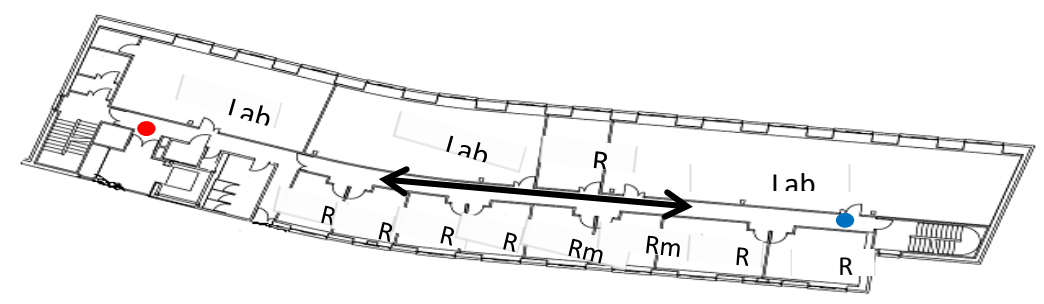

Fig.2: detailed map of signal propagation at the corridor

\subsection{Results and Discussion}

In order to predict the pathloss model for the environment under consideration at $24 \mathrm{GHz}$ spectrum, the values for predicted pathloss (UL) are calculated using (2) and (3), the pathloss exponent $(n)$ is derived from the measured values using Linear Regression concept to minimize the difference between the measured and predicted pathloss values means square error, and to compute the values of $\mathrm{n}$ and $\sigma[20]$ as represented by the following equations:

$$
\mathrm{e}(\mathrm{n})=\sum_{k=1}^{m}(P L-U L)^{2}
$$

The computation procedures are as simplified in the table below: 
Table 1: Computation of Mean square error

\begin{tabular}{|c|c|c|c|c|}
\hline Distance(m) & $P L(d B)$ & $\mathrm{UL}(\mathrm{dB})$ & (PL-UL)dB & $(\mathrm{PL}-\mathrm{UL})^{2} \mathrm{~dB}$ \\
\hline 1 & 54.033 & 54.033 & 0 & $0-$ \\
\hline 6 & 56.37 & $54.03+7.7 n$ & $2.33-7.7 n$ & $\begin{array}{c}5.43- \\
35.88 n+59.29 n^{2}\end{array}$ \\
\hline 11 & 63.46 & $54.03+10.04 n$ & $9.43-10.04 n$ & $\begin{array}{c}88.93- \\
189.35 n+100.80 n^{2}\end{array}$ \\
\hline 16 & 68.09 & $54.03+12.04 n$ & $13.72-12.04 n$ & $\begin{array}{c}188.23- \\
330.37 n+144.96 n^{2}\end{array}$ \\
\hline 21 & 69.03 & $54.03+13.22 n$ & $\begin{array}{l}14.66- \\
13.22 \mathrm{n}\end{array}$ & $\begin{array}{c}214.91- \\
387.61+174.76 \mathrm{n}^{2}\end{array}$ \\
\hline 26 & 73.26 & $54.03+14.14 n$ & $\begin{array}{l}18.89- \\
14.14 \mathrm{n}\end{array}$ & $\begin{array}{c}356.83- \\
534.20 n+199.94 n^{2}\end{array}$ \\
\hline 31 & 79.91 & $54.03+14.91 n$ & $\begin{array}{l}25.54- \\
14.91 \mathrm{n} \\
\end{array}$ & $\begin{array}{c}652.29- \\
761.60 n+222.31 n^{2} \\
\end{array}$ \\
\hline 36 & 82.12 & $54.03+15.56 n$ & $\begin{array}{c}27.75- \\
15.56 \mathrm{n} \\
\end{array}$ & $\begin{array}{c}770.06- \\
863.5 n+242.22 n^{2} \\
\end{array}$ \\
\hline
\end{tabular}

From the table,

e (n) $=1144.17 n 2-3100.51 n+2276.68$

By differentiate (7), the value of $n$ was computed as;

$\mathrm{n}=1.4$

The standard deviation was derived in a similar manner using:

$\sigma(d B)=\sum_{k=1}^{m} \sqrt{\frac{(P L-U L)^{2}}{m}}$

$m=8$, is the number of locations where measurements were conducted

$\sigma(\mathrm{dB})=4.9 \mathrm{~dB}$ 
The model for the considered propagation environment using (4) and the calculated parameters therefore is,

$P L(d B)=54.03+10(1.4) \log (d)+4.9$

$P L(d B)=54.02+14 \log (d)+4.9$

The results of the experiments yielded the path loss exponential of 1.4 and standard deviation of $4.9 \mathrm{~dB}$. Since the path loss falls within the estimated values in such scenario (1.2-8) [3], then it can be concluded that the predicted mathematical model for the described environment is accurate. Also the low value of the path loss suggests that the signal loss in this scenario is low. This peculiarity can be traced to the wave guiding effects that enhance the signal propagation in the corridor. Since the predicted path loss is lower than the free space propagation path loss, aggregate throughput of data rate is high, hence improved system performance is achieved.

The equation generated from pathloss prediction is used to create the models shown Figs 13 , to visualize the propagation phenomenon for the propagation environment. The Figs reveal that the measurements models outperformed both the free space model and proposed model by an average of $10 \mathrm{~dB}$ and $8 \mathrm{~dB}$ respectively, while a convergence is seen between the measure and predicted models as the distance increases (Fig 4).The predicted models though outperformed the free space model, both are seen to be almost same the at the initial stage but deviate as the distance increases with an average difference of $10 \mathrm{~dB}$ at $36 \mathrm{~m}$ distance (Fig 5).

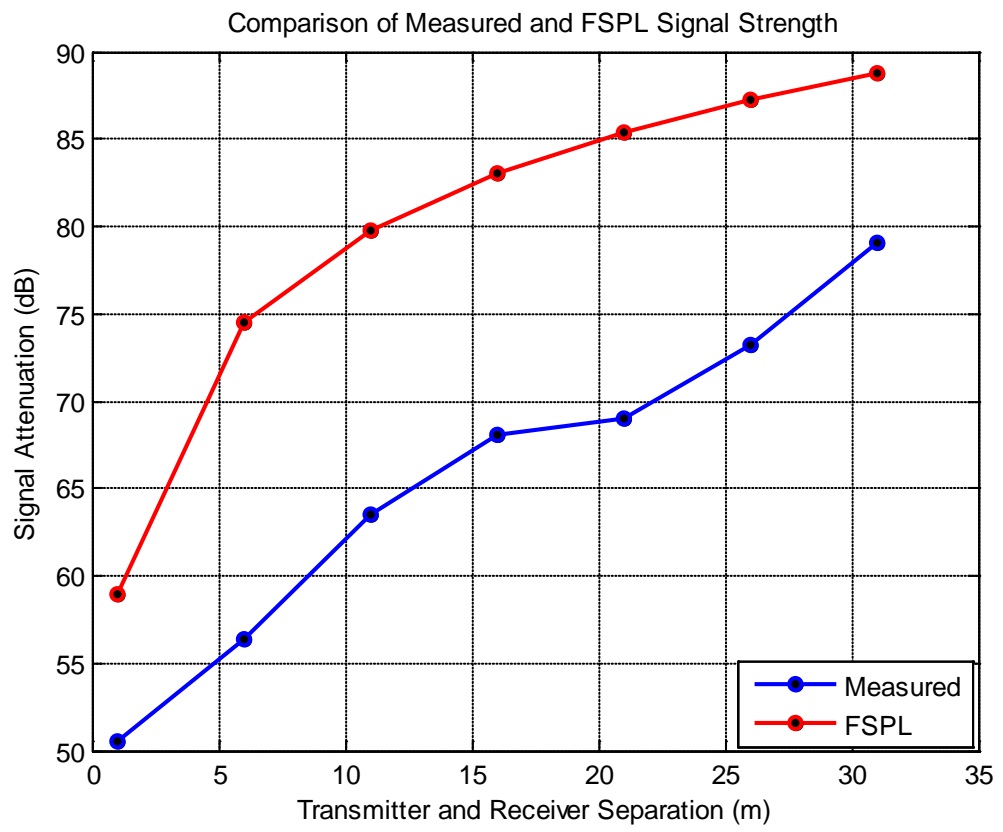

Fig 3: Measured Signal Pathloss and FSPL compared 


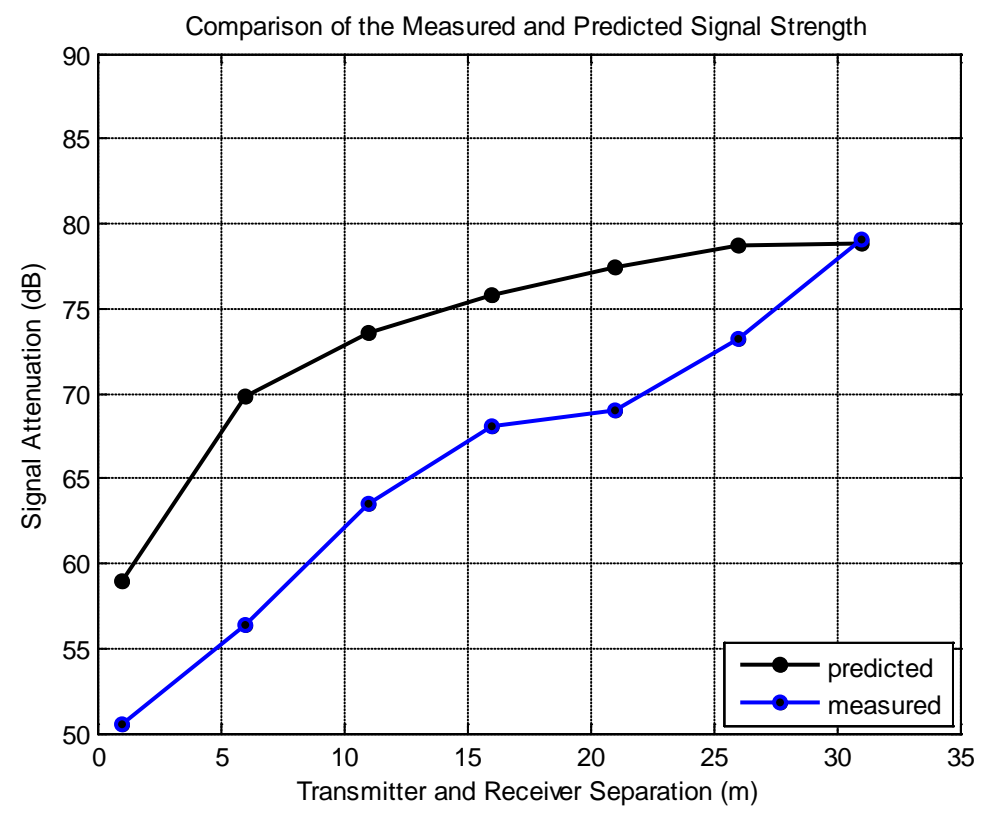

Fig 4: Measured and Predicted Signal Pathloss compared

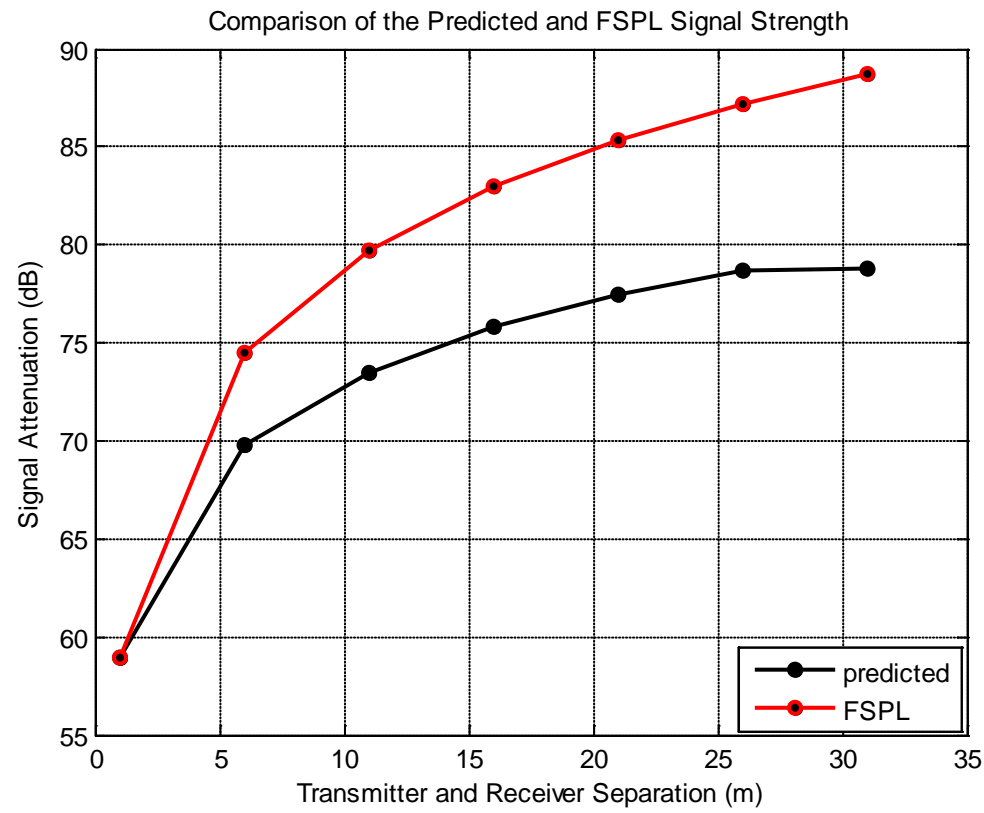

Fig 5: Predicted Signal Pathloss and FSPL compared

\section{Conclusion}

In this work, indoor propagation model was developed for a corridor based on empirical model of Log-distance path loss model and log-normal shadowing. The path loss equation for our scenario was determined through numerical analysis of measurement results. The results of the experiments yielded the path loss exponential of 1.4 and standard deviation of $4.9 \mathrm{~dB}$. The pathloss exponent of 1.4 realized through the pathloss prediction empirical analysis is 
significant: Since the path loss falls within the estimated values in such scenario (1.2-8), then it can be concluded that the predicted mathematical model for the described environment is accurate. Its low value confirmed the good signal strength achieved in this scenario during wireless transmission as reported in earlier work [25], establishing the fact that the waveguidelike effects of the corridor enhanced the signal throughput through reflection; it also represents the different obstruction the signal passed through during propagation. Since the predicted path loss is lower than the free space propagation path loss, aggregate throughput of data rate is high, hence improved system performance is achieved. The results show that a hallway/corridor as well as typical office can be flooded with gigabit/s through wireless transmission to enable seamless communication and adequate bandwidth requirement for multimedia applications services.

\section{REFERENCES}

[1]. Xingang G, Sumit R, and W. S. Conner, Spatial reuse in wireless ad-hoc networks, in Vehicular Technolohg Conference, 2003. VTC 2003-Fall. 2003 IEEE 58 ${ }^{\text {th }}$.IEEE 2003, vol. 3 pp. 1437-1442 .

[2]. Yann L, et al, Beamforming techniques for enabling spatial-reuse in MCCA 802.11 s networks, EURASIP Journal on Wireless Communications and Networking, vol. 2011, pp. 1-13, 2011.

[3]. Andrej $\mathrm{H}$, et al, A Survey of Radio Propagation Modeling for Tunnels., IEEE Communications Surveys and Tutorials, vol. 16, no. 2, pp. 658-669, IEEE 2013

[4]. Youngmoon K, et al, Analysis of radio wave propagation characteristics in rectangular road tunnel at 800 $\mathrm{MHz}$ and 2.4 GHz, in Antennas and Propagation Society International Symposium, 2003. IEEE, 2003, vol. 3, pp. 1016-1019.

[5]. CG Liu, et al, Modelling radio wave propagation in tunnels with ray-tracing method, in Antennas and Propagation (EuCAP), 2013 7th European Conference on IEEE, 2013, pp. 2317-2321.

[6]. Yue P. Z, Novel model for propagation loss prediction in tunnels, Vehicular Technology, IEEE Transactions on, vol. 52, pp. 1308-1314, 2003.

[7]. Jadhavar R and Sontakke TR, $2.4 \mathrm{GHz}$ Propagation Prediction Models for Indoor Wireless Communications Within Building, International Journal of Soft Computing and Engineering (IJSCE), vol. 2, pp. 108-113, 2012.

[8]. Pengfei $X$, et al, Short range gigabit wireless communications systems: potentials, challenges and techniques, in Ultra-Wideband, 2007. ICUWB 2007. IEEE International Conference on IEEE, 2007, pp. 123-128.

[9]. Dai L and Dave R, Investigation of indoor radio channels from $2.4 \mathrm{GHz}$ to $24 \mathrm{GHz}$, in Antennas and Propagation Society International Symposium, 2003. IEEE, 2003, pp. 134-137.

[10]. Dia L and David R, Indoor wireless channel modeling from 2.4 to $24 \mathrm{GHz}$ using a combined E/H-Plane 2D ray tracing method, in Antennas and Propagation Society International Symposium, 2004. IEEE, 2004, pp. 36413644.

[11]. John C. S, Indoor radio WLAN performance part II: Range performance in a dense office environment, Intersil Corporation, 1998. 
[12]. Ali-Rantala $P$, et al, Different kinds of walls and their effect on the attenuation of radiowaves indoors, in Antennas and Propagation Society International Symposium, 2003. IEEE, 2003, vol. 3 pp. 1020-1023.

[13]. Robert G A, et al,Indoor propagation modeling at $2.4 \mathrm{GHz}$ for IEEE 802.11 networks, International Association of Science and Technology for Development, 2005.

[14]. I. Rosu, Basics of Radio Wave Propagation. YO3DAC/VA3IUL, 10.

[15]. Jim Z and AI P, Tutorial on basic link budget analysis, Application Note AN9804, Harris Semiconductor, 1998.

[16]. Aleksandar N, et al, Modern approaches in modeling of mobile radio systems propagation environment, Communications Surveys \& Tutorials, IEEE, vol. 3, pp. 2-12, 2000.

[17]. Muzaiyanah $\mathrm{H}$, et al, Wifi signal propagation at $2.4 \mathrm{GHz}$, in Microwave Conference, 2009. APMC 2009. Asia Pacific, 2009, pp. 528-531.

[18]. Iskander M. F. and Yun Z., Propagation prediction models for wireless communication systems, Microwave Theory and Techniques, IEEE Transactions on, vol. 50, pp. 662-673, 2002.

[19]. Jorgen B A, et al, Propagation measurements and models for wireless communications channels, Communications Magazine, IEEE, vol. 33, pp. 42-49, 1995.

[20]. Scott Y S and Theodore S R, $914 \mathrm{MHz}$ path loss prediction models for indoor wireless communications in multifloored buildings, Antennas and Propagation, IEEE Transactions on, vol. 40, pp. 207-217, 1992.

[21]. Cebula S, et al, Empirical channel model for $2.4 \mathrm{GHz}$ ieee 802.11 wlan, in Proceedings of the 2011 International Conference on Wireless Networks, 2011.

[22]. Halford K. and Webster M, Multipath measurement in wireless LANs, Intersil Application Note AN9895, vol. $1,2001$.

[23]. Yun S., et al., Hybrid division duplex system for next-generation cellular services, Vehicular Technology, IEEE Transactions on, vol. 56, pp. 3040-3059, 2007.

[24]. Sang Y. J., et al An Overlaid Hybrid-Division Duplex OFDMA System with Multihop Transmission, ETRI Journal, vol. 33, p. 201, 2011.

[25]. Femi-Jemilohun O.J, et al, An Experimental Investigation into GbE Wireless Data Communication at $24 \mathrm{GHz}$ in Non-Line-of-Sight and Multi-path Rich Environments in IEEE Antenna and Wireless Propagation Letters, 2014, vol. 13 pp1219-1222, 